\title{
Manejo anestésico para cirugía de estimulación cerebral profunda en la enfermedad de Parkinson
}

\author{
Anesthetic management for deep brain stimulation \\ electrode placement surgery in Parkinson's disease
}

Silvina Longo ${ }^{1}$, Fernando Dominella², Ana Lucía Arnaiz², Luis Masco²

\begin{abstract}
Parkinson's disease is highly prevalent and one of its treatments in pursue of improving quality of life is deep brain stimulation of deep basal ganglia. Although the anesthetic management varies, we consider that the most appropriate approach with respect to risk/benefit ratio is the asleep/awake/ asleep technique, since it allows the interaction of the patient with the multidisciplinary team in order to guarantee a correct placement of the stimulation electrodes. We present a 10 case-series of patients where we describe the use of dexmedetomidine as the main drug to achieve the desired depth of anesthesia, ensuring collaboration during neurological check-up. The anesthesiologist as part of the neurosurgical team has an active role in achieving adequate levels of sedation, analgesia and awareness when needed during the different stages of this procedure, where dexmedetomidine has proven to be a fundamental pillar in this work to achieve the objectives.
\end{abstract}

\section{RESUMEN}

La enfemedad de Parkinson es prevalente y uno de sus tratamientos en busca de mejorar la calidad de vida es la estimulación cerebral profunda. Si bien el manejo anestésico es variado, consideramos que el más adecuado respecto a los riesgos/beneficios es la técnica dormido/despierto/dormido, ya que permite la interacción del paciente con el equipo multidisciplinario para garantizar una

\section{Key words:}

Parkinson,

conscious sedation, anesthesia asleep/awake/ asleep,

dexmedetomidine

\section{Palabras clave:}

Parkinson,

anestesia dormido/ despierto/dormido, dexmedetomidina

\section{Anestesióloga.}

2 médicos residentes de anestesiología.

Hospital Privado Universitario de Córdoba, Argentina.

Fecha de recepción: 12 de septiembre de 2020

Fecha de aceptación: 25 de noviembre de 2020

\section{ORCID}

0000-0001-5822-8727

Correspondencia:

Dra. Silvina Longo

Silvina.longo@icloud.com 
correcta colocación de los electrodos de estimulación. Presentamos una serie de casos, 10 pacientes donde describimos la utilización de dexmedetomidina como droga principal para lograr la colaboración durante el chequeo neurológico. La dexmedetomidina ha sido en este trabajo un pilar fundamental para lograr los objetivos. El anestesiólogo como parte del equipo neuroquirúrgico tiene un rol activo, logrando adecuados planos de anestesia y analgesia durantes las diferentes etapas de la cirugía.

\section{Introducción}

L a enfermedad de Parkinson (EP) es una enfermedad neurodegenerativa prevalente en nuestro medio que se caracteriza por una pérdida de neuronas dopaminérgicas en la sustancia negra del ganglio basal y una disminución en la producción de dopamina[1].

El diagnóstico y las características clínicas de la enfermedad de Parkinson son: temblor de reposo, rigidez muscular, bradicinecia, y pérdida de reflejos posturales. El tratamiento incluye el uso de L-dopa o agonistas de los receptores de dopamina. Algunos pacientes reciben anticolinérgicos que interfieren con la producción o la captación del neurotransmisor acetilcolina. Estos medicamentos ayudan a reducir el temblor y la rigidez muscular. Los pacientes con EP reciben muchas veces antidepresivos, debido a la alta incidencia de esta enfermedad en ellos.

El tratamiento, además de las medidas farmacológicas, incluye rehabilitación fisioterapéutica y estimulación psiconeurocognitiva. Otra alternativa para mejorar la calidad de vida, es la colocación de electrodos intracerebrales (técnica de Estimulación Cerebral Profunda (ECP). Ésta se utiliza para corregir la actividad eléctrica anormal del cerebro que causa trastornos del movimiento. Su principal indicación es en pacientes que tienen síntomas que no se controlan con medicamentos. La ECP resulta más segura y efectiva que las antiguas intervenciones quirúrgicas para trastornos del movimiento, que dejaban lesiones en el cerebro[2],[3].

La ECP es un procedimiento mínimamente invasivo que coloca electrodos en lugares específicos del cerebro para realizar pruebas clínicas, los cuales son posteriormente conectados a un marcapasos implantado, llamado estimulador (E)[4].

\section{Materiales y Métodos}

Describimos 10 pacientes que se sometieron a procedimiento de colocación de ECP en nuestra institución. Las edades fueron entre 50 y 78 años. Todos los pacientes fueron ASA 2 en la valoración preoperatoria; la principal comorbilidad hallada fue la hipertensión arterial (en 7 pacientes), 3 tenían diabetes tipo 2, y 1 paciente con obesidad moderada y sindrome de apnea obstructiva del sueño (SAOS).

Se obtuvo el consentimiento informado en todos los pacientes.

Se utilizó la técnica anestesiado/despierto/anestesiado, en la cual el paciente recibe un inductor endovenoso para lograr hipnosis y sedación.

En el quirófano se posiciona al paciente en decúbito dorsal, teniendo en cuenta el confort del paciente. Se canaliza con vía periférica. Se utiliza monitoreo no invasivo: presión arterial, cardioscopía, frecuencia respiratoria, oxímetro de pulso, índice biespectral (BIS), temperatura y se administra $\mathrm{O}_{2}$ suplementario por canula a 3 litros $x$ min.

Se inicia infusión de dexmedetomidina con dosis de carga, en todos los pacientes, $1 \mathrm{mcg} / \mathrm{kg}$ a pasar en $10 \mathrm{~min}$, luego dosis de mantenimiento a 0,3 a 0,5 $\mathrm{mcg} / \mathrm{kg} / \mathrm{h}$. Durante la trepanación se logra un plano de anestesia general con propofol 1 a $2 \mathrm{mgr} / \mathrm{kg}$, y luego al final del procedimiento, anestesia general con intubación orotraqueal para la colocación del estimulador en el tórax.

El paciente participa del procedimiento quirúrgico y colabora con el equipo durante la etapa intermedia de la cirugía. Resulta fundamental realizar una adecuada anestesia local del sitio quirúrgico, la cual es llevada a cabo por el neurocirujano mediante bloqueo del nervio supraorbitario con lidocaina $2 \%$ e infiltración del colgajo.

\section{Desarrollo del procedimiento quirúrgico-anesté- sico}

El éxito en la cirugía de ECP comienza con la selección cuidadosa del paciente durante la evaluación preoperatoria. Analizando la condición física general del paciente y la función neurocognitiva. La misma se 
lleva a cabo por un equipo multidisciplinario de anestesiólogos, neurólogos, neurocirujanos y neuropsicólogos para garantizar que se evalúan todos los aspectos del cuidado del paciente.

Manejo perioperatorio de medicamentos para EP: Se debe considerar que la suspensión brusca de algunos fármacos puede provocar la excerbación de la patología de base, o síndrome de retirada. Algunos ejemplos de fármacos que producen este efecto son los bloqueantes $\beta$-adrenérgicos, los agonistas $\alpha$-adrenérgicos, los antidepresivos inhibidores de la monoaminooxidasa, los antidepresivos tricíclicos, las benzodiacepinas, los antiepilépticos y los antiparkinsonianos. En general las complicaciones se resuelven reintroduciendo la medicación habitual[5],[6].

Se debe revisar en forma cuidadosa el regimen de antiagregación plaquetaria o anticoagulación (si es que el paciente los presenta) con el fin de evitar complicaciones hemorrágicas perioratorias. Para medicamentos específicos de la enfermedad, instrucciones claras para la continuación o interrupción deben informarse a los pacientes a discreción del equipo multidisciplinario, ya que algunos pacientes tienen que estar en un estado de "libre de fármacos" para realizar el mapeo intraoperatorio y las pruebas clínicas. Si los síntomas son severos, una dosis reducida de su medicación habitual puede ser una opción alternativa. En todos nuestros pacientes se suspendió la dosis de la noche previa, con el fin de monitorizar más efectivamente la respuesta a la estimulación cerebral.

Además, de garantizar que el paciente se encuentre médicamente optimizado para el procedimiento, debe considerarse que los pacientes con EP comúnmente sufren de apnea obstructiva del sueño o tienen un mayor riesgo de aspiración. Su precisa evaluación es importante al considerar cualquier técnica de sedación. Las alternativas para asegurar la vía aérea en cualquier etapa del procedimiento deben haberse preparado de antemano[7].

La cooperación del paciente durante el período despierto de la cirugía es imprescindible para el éxito. La identificación de factores que pueden complicar esto, tales como retraso del desarrollo, demencia, dificultades de comunicación, claustrofobia o dificultad previa con la sedación, requieren atención especial. Es fundamental que durante la visita preoperatoria se brinde una explicación detallada del procedimiento para tranquilizar al paciente, aliviar la ansiedad, identificar posibles dificultades intraoperatorias y mejorar la cooperación durante la cirugía.

\section{Primera Etapa:}

Un marco estereotáxico se fijó en la cabeza del paciente, con anestesia local el día previo a la cirugía (Figura1). Una vez colocado el marco, se realiza una exploración con tomografía axial computada cerebral con el fin de establecer las coordenadas para la colocación exacta de los electrodos.

\section{Segunda etapa:}

Intraoperatoriamente, se colocan 3 litros de oxígeno mediante una cánula nasal. Logramos una adecuada temperatura y posición para asegurar la comodidad del paciente. La colocación de campos quirúrgicos, nos permitió el acceso a la cara, brazos y piernas del paciente, mantener la esterilidad y evitar la acumulación de dióxido de carbono. Mantuvimos escasa administración de líquidos, que es esencial para evitar la excesiva distensión de la vejiga y el consiguiente disconfort del paciente.

Se comienza con la carga de dexmedetomidina durante $10 \mathrm{~min}, 1 \mathrm{ug} / \mathrm{kg}$ de fentanilo y lidocaina 1 $\mathrm{mg} / \mathrm{kg}$, ketorolac $30 \mathrm{~m}$ y dexametasona $8 \mathrm{~m}$. El cirujano realiza anestesia local del sitio quirúrgico y en el momento a la trepanación del primer lado, se utiliza propofol 1 a $2 \mathrm{mg} / \mathrm{kg}$, ya que éste es el momento de mayor estimulación quirúrgica. Procedimiento que se repite al momento de la segunda trepanación.

Luego se insertan los electrodos de estimulación a través de esos orificios (Figuras 2 a y b).

Todos los pacientes conservaron la capacidad de

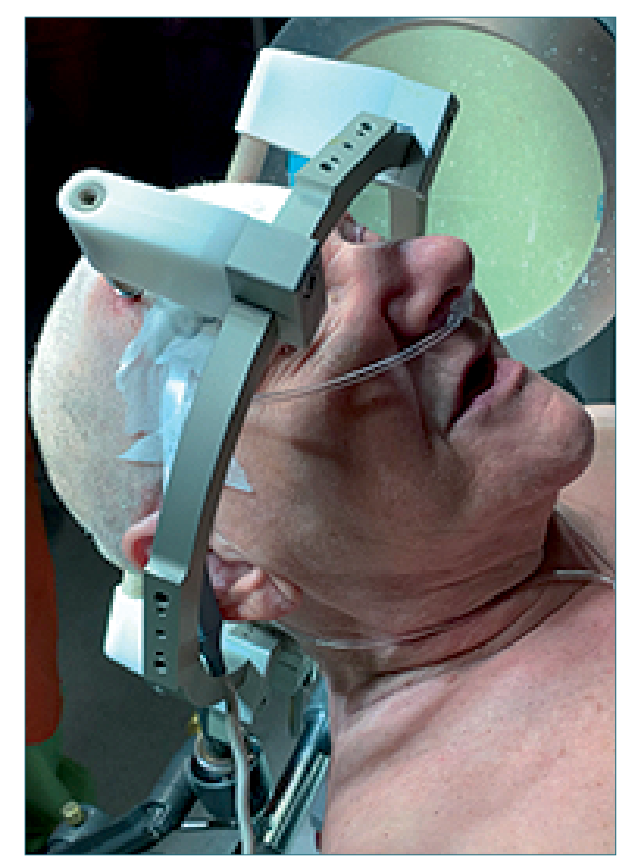

Figura 1. Marco estereotáxico. 


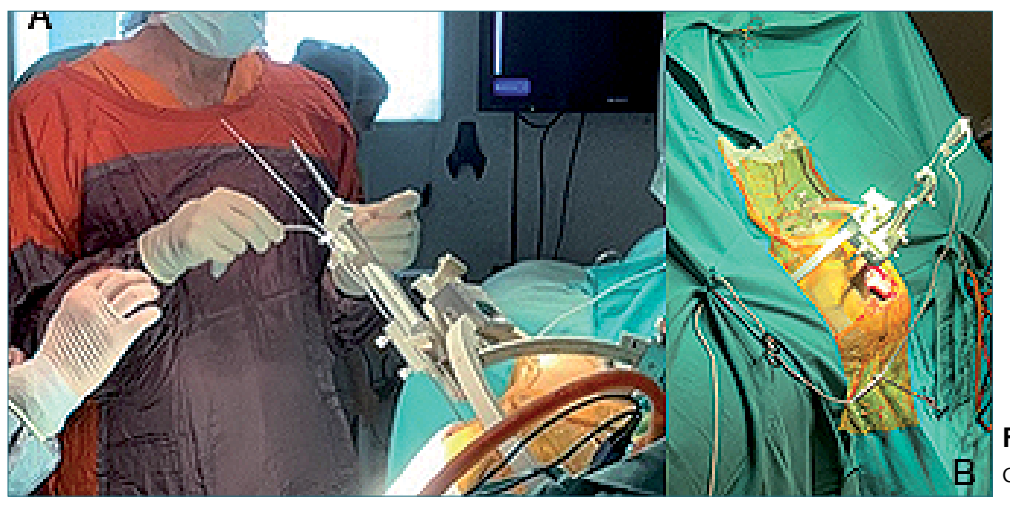

Figuras 2. a: Inseción de electrodos por craneotomía; b: Cables de electrodo.s

interactuar con el equipo quirúrgico y toleraron adecuadamente el procedimiento. Durante este período el paciente bajo mínima sedoanalgesia, en rango de BIS entre 75 y 90, los pacientes fueron capaces de hablar claramente con el neurólogo y anestesiólogo repitiendo su nombre y apellido, contando hasta 10 , movilizando dedos de la mano y los pies, dirigiendo la mirada hacia un lado y otro (Figura 3). Este paso fue esencial para facilitar la localización del microelectrodo, y las pruebas clínicas ayudaron a confirmar el posicionamiento óptimo del electrodo y la detección de efectos secundarios. Logramos la colaboración del paciente durante el mapeo neurofisiológico en busca de los puntos de descarga (temblor distal), para lo cual la dexmedetomidina fue especialmente útil por impedir la modificación de los registros neurofisiológicos[8] (Figura 4 a y b),

Con esta técnica logramos mantener al paciente hemodinámicamente estable (Figura 5).

Si bien no fue necesario en ningún caso, es importante que durante el procedimiento, estén disponibles las herramientas necesarias para extraer el marco principal para permitir el acceso de emergencia a la vía aérea.

\section{Tercera etapa:}

Los electrodos se conectaron a un cable externo que luego fue tunelizado por vía subcutánea bajo el cuero cabelludo, la nuca, y finalmente al sitio de la implantación del generador de pulso implantable. El E fue colocado en la pared del tórax bajo anestesia general endovenosa, se realizó intubación orotraqual y se emplearon dexmedetomidina, propofol, remifentanilo y rocuronio.

La observación neurológica postoperatoria es necesaria luego del procedimiento, ya que es un procedimiento intracraneal. Los medicamentos antiparkinsonianos deben ser reanudados tan pronto como sea

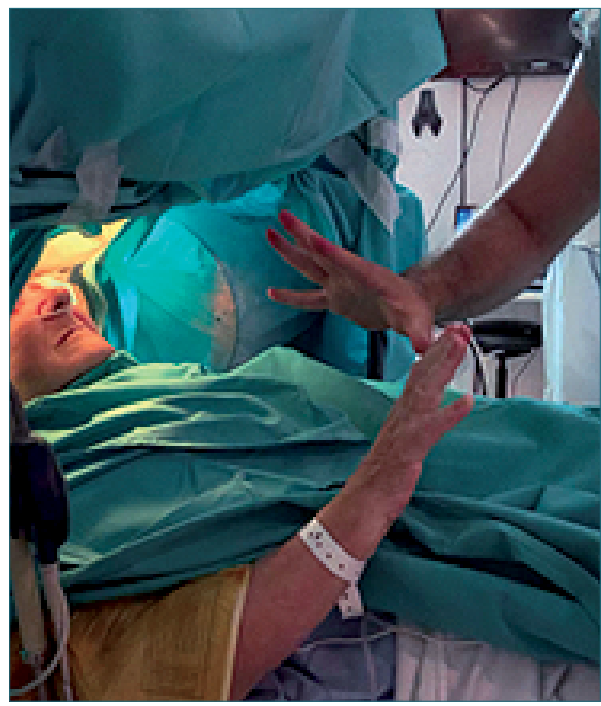

Figura 3. Respuesta de la paciente a las órdenes del neurólogo.

posible, para evitar el deterioro en la función motora y neurológica. Los pacientes generalmente requieren asistencia con las actividades diarias las siguientes semanas a la inserción.

\section{Resultados}

Se incluyeron 10 pacientes (6 mujeres y 4 hombres), cuyas edades oscilaron de 50 a 78 años, todos recibieron apoyo no invasivo de la ventilación con oxígeno por cánulas nasales, el tiempo quirúrgico fue de $5 \pm 1 \mathrm{~h}$, las comorbilidades de los pacientes fueron además de EP, hipertensión arterial, diabetes tipo 2, hipotiroidismo y 1 solo paciente obesidad moderadada y SAOS. 


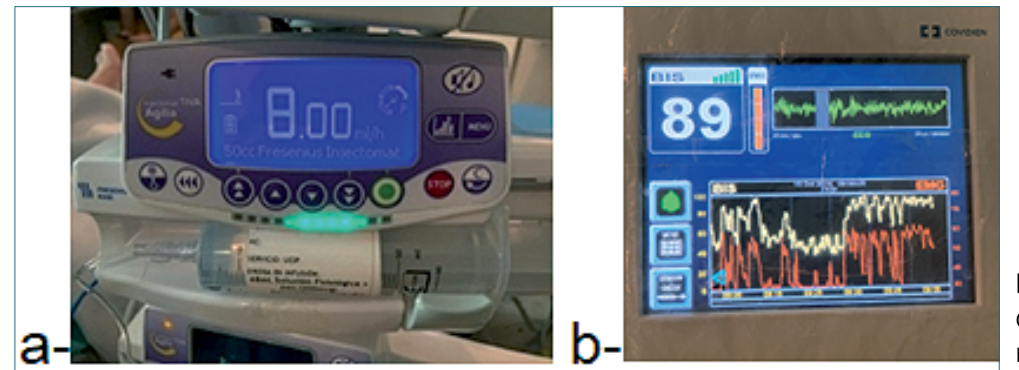

Figura 4. a: Infusión de dexmedetomidina; b: BIS durante las respuestas neurológicas.

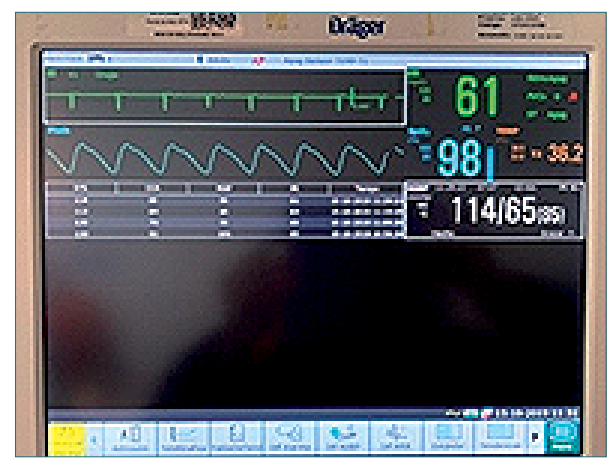

Figura 5. Monitoreo, con adecuadas condiciones hemodinámicas.

Todos los pacientes mantuvieron adecuada tensión arterial. La frecuencia cardíaca bajo en $20 \%$ luego de la infusión del bolo inicial de dexmedetomidina, para luego recuperarse al 10\% menor del valor basal a lo largo de la cirugía.

Todos los pacientes colaboraron con el procedimiento quirúrgico, obedeciendo a las siguientes órdenes: repetición de su nombre y apellido, movimientos dedo-nariz, mímica y expresión facial, repetir números y palabras cortas y movimiento de los dedos y las piernas. Se valoró el dolor con la escala visual análoga (EVA), los pacientes no requirieron analgesia adicional. No se registraron efectos colaterales adversos. Se logró un adecuado comportamiento durante la etapa despierto, todos los pacientes pudieron colaborar adecuadamente y sin exitación.

En un paciente con antecedentes de SAOS obsevamos hipercapnia que se detectó y resolvió en la última etapa del procedimiento al realizar anestesia general con intubación orotraqueal. Posiblemente debido a la falta de disponibilidad de monitarizar con capnometría durante la sedación.

Durante la visita post operatoria la mayoría de los pacientes no recordaba exactamente los momentos del despertar, manifestaron que se volverían a some- terse al procedimiento en las mismas condiciones. Se manifestaron felices y con alta expectativa hacia el futuro.

\section{Discusión}

La ECP es un procedimiento en expansión para tratar la EP ya que puede lograr que estos pacientes mejoraren su calidad de vida. Comunmente, se utiliza la técnica despierto y sedado, debido a que es necesario realizar pruebas neurológicas intraoperatorias para faciliar el mapeo neurofisiológico y disminuir las complicaciones.

La ECP es generalmente muy segura pero, como con toda cirugía, hay riesgos perioperatorios y complicaciones. Una pequeña serie de casos identifica una tasa de complicación intraoperatoria de aproximadamente $7 \%$. Las principales complicaciones incluyen sangrado intracraneal $(0,4 \%-3,6 \%)$, convulsiones $(0,8 \%-4,5 \%)$, ACV, déficit neurológico $(0,3 \%-0,6 \%)$ y delirio posterior al procedimiento[9]. Otras complicaciones intraoperatorias incluyen obstrucción de la vía aérea (1,6\%-5,5\%), hipertensión, hipotensión o embolia aérea venosa (1,6\%-3,5\%)[10],[11]. Complicaciones relacionadas con el dispositivo en sí mismo incluyen infección, falla y migración del electrodo.

La dexmedetomidina es un agente excelente para lograr adecuada sedoanalgesia y le permite al paciente responder órdenes. La adecuada preparación del paciente y la efectiva comunicación del equipo es fundamental. El anestesiólogo logra en este tipo de cirugía un rol activo.

Se ha demostrado que la dexmedetomidina proporciona una sedación exitosa sin deterioro de la monitorización electrofisiológica en neurocirugía funcional[12],[13].

Si bien no registramos complicaciones por el uso de dexmedetomidina en nuestra serie de casos, hay algunas consideraciones importantes adicionales para el uso de esta droga. Primero, se sabe que la dex- 
medetomidina causa hipotensión y esta respuesta puede aumentarse en pacientes con enfermedad de Parkinson. Los anestesiólogos deben estar preparados para esta respuesta potencial y, además, preparados para el inevitable aumento de la presión arterial una vez que se suspenden las infusiones. Además, en un ensayo clínico controlado demostraron un aumento en la incidencia de algunos eventos adversos como la agitación paradójica (14\% de incidencia a tasas de infusión > 1,1 $\mu \mathrm{g} / \mathrm{kg} / \mathrm{h}$ ) a dosis más altas[14]. Este evento adverso particular puede ser potencialmente peligroso en un paciente fijado a la mesa de la sala de operaciones en el marco de la cabeza. Por lo tanto, es necesario conocer y reconocer esta posible reacción adversa, así como un plan alternativo para la sedación consciente, especialmente en pacientes que requieren infusiones de dosis más altas.

Se ha informado de una diversidad de técnicas para proporcionar buenas condiciones operativas en el estado de vigilia mientras se mantiene la vía aérea. La anestesia ha variado desde sedación consciente utilizando propofol y/o dexmedetomidina, junto con pequeñas cantidades de remifentanilo, hasta anestesia general con intubación endotraqueal, utilizando agentes endovenosos o de inhalación[15],[16]. Las dos técnicas principales de sedación consciente son dormido-despierto-dormido (DDD) y cuidados anestésica monitorizada (CAM) con sedación[17]. En cualquier caso, las técnicas de vigilia proporcionan las mejores condiciones para la neurofisiología intraoperatoria y las pruebas de estimulación. Las técnicas de sedación consciente, con todos los medicamentos suspendidos durante al menos 15 minutos antes de la estimulación, proporcionan los datos más confiables para ayudar a la colocación precisa de los electrodos[18].

Se ha informado que dexmedetomidina causa un mínimo de problemas de depresión respiratoria en voluntarios sanos y pacientes sin enfermedades respiratorias, y esto es consistente con nuestro serie actual. Sin embargo, un paciente con antecedentes de SAOS desarrolló hipercapnia durante la infusión de la droga. Esto es no es sorprendente, ya que este fármaco es un sedante. Aunque lo hace, no causó depresión respiratoria. Dexmedetomidina puede provocar obstrucción de la vía aerea superior por pérdida del tono muscular incluso en pacientes sin SAOS.

\section{Conclusiones}

La ECP se está convirtiendo en un procedimiento cada vez más frecuente. El manejo anestésico constituye un desafío para los profesionales ya que la técnica dormido/despierto/dormido no es habitualmente realizada. El anestesiólogo ocupa un lugar fundamental durante el trabajo en equipo que busca mejorar la calidad de vida de los pacientes con EP.

La dexmedetomidina es un agente comúnmente utilizado[19],[20],[21]. Proporciona sedación con mínima depresión respiratoria y atenúa las variaciones hemodinámicas intraoperatorias. El propofol es también comúnmente empleado durante la colocación de electrodos. El propofol puede causar discinesia y abolir el temblor[22]. Los opioides de acción corta tienen un efecto mínimo sobre los efectos de los microelectrodos, pero dosis altas pueden causar empeoramiento de la rigidez. Las benzodiazepinas no se recomiendan ya que pueden abolir la respuesta a los electrodos e interferir con las pruebas de estimulación[23]. El régimen de sedación usado logró el objetivo de minimizar cualquier efecto sobre la actividad subcortical, optimizando así el registro de los microelectrodos y las pruebas clínicas (Figura 5).

Con la sedación consciente con dexmedetomidina logramos un adecuado grado de sedación, los pacientes cumplieron las siguientes órdenes: movimientos de manos y pies, mímica y expresión facial, repetir números y nominar[24],[25],[26],[27],[28]. No ocurrió depresión respiratoria, ni otro tipo de complicaciones. Se logró además un adecuado plano de analgesia/ anestesia, sin causar molestias en el paciente.

\section{Referencias}

1. Ministerio de Salud de la Republica Argentina. "Estudios de carga de enfermedad". 2018

2. Miocinovic $S$, Somayajula $S$, Chitnis $S$, et al. History, applications and mechanisms of deep brain stimulation. JAMA Neurol 2013;70:163-171. https://doi. org/10.1001/2013.jamaneurol. 45

3. Alkhani A, Lozano AM. Pallidotomy for Parkinson disease: A review of contemporary literature. J Neurosurg 2001;94:43-
9. https://doi.org/10.3171/ jns.2001.94.1.0043

4. Krack P, Batir A, Van Blercom $N$, et al. Five-year follow-up of bilateral stimulation of the subthalamic nucleus in advanced Parkinson's disease. N Engl J Med 2003;349:1925-34. https://doi. 
org/10.1056/NEJMoa035275

5. Nicholson G, Pereira AC, Hall GM. Parkinson's disease and anaesthesia. Br J Anaesth 2002;89:904-16. https://doi. org/10.1093/bja/aef268

6. Juvany Roig R., Mercadal Orfila G., Jódar Maanés R. Manejo perioperatorio de la medicación crónica no relacionada con la cirugía 293- 294 AN. MED. INTERNA Vol. 21, N.o 6, 2004 (Madrid) https://doi.org/10.4321/ S0212-71992004000600009

7. Ard J, Doyle W, Bekker A. Dexmedetomidine in awake craniotomy: A technical note. Surg Neurol 2005;63:114 -7. https://doi.org/10.1016/j.surneu.2004.02.029

8. Gorgulho A., De Salles A.A.F., Frighetto L., Behnke E. Incidence of hemorrhage associated with electrophysiological studies performed using macroelectrodes and microelectrodes in functional neurosurgery. J Neurosurg 2005; 102:888 -96. https://doi.org/10.3171/ jns.2005.102.5.0888

9. Binder DK, Rau GF, Starr PA. Risk factors for hemorrhage during microelectrodeguided deep brain stimulator implantation for movement disorders. Neurosurgery 2005;56:722-32. https://doi.org/10.1227/01. NEU.0000156473.57196.7E

10. Khatib, Reem, Ebrahim, Zeyd, Rezai, Ali, Cata, Juan, Boulis, Nicolas, John Doyle, D., MD, PhD, Schurigyn, Tamara, CRNA, MSN, Farag, Ehab, MD, FRCA Perioperative Events During Deep BrainStimulation: The Experience at Cleveland Clinic. Journal of Neurosurgical Anesthesiology January 2008:20:1. https://doi.org/10.1097/ ANA.0b013e318157a15a

11. Venkatraghavan L, Manninen P. Anesthesia for deep brain stimulation. Curr Opin
Anaesthesiol. 2011; 24:495499. https://doi.org/10.1097/ ACO.0b013e32834a894c

12. Erickson KM, Cole DJ. Anesthetic considerations for awake craniotomy for epilepsy and functional neurosurgery. Anesthesiol Clin. 2012; 30:241268. https://doi.org/10.1016/j. anclin.2012.05.002

13. Rozet I. Anesthesia for functional neurosurgery: the role of dexmedetomidine. Current Opinion in Anaesthesiology 2008, 21:537543. https://doi.org/10.1097/ ACO.0b013e32830edafd

14. Ebert TJ, Hall JE, Barney JA, Uhrich TD, Colinco MD. The effects of increasing plasma concentrations of dexmedetomidina in humans. Anesthesiology 2000;93:382-94. https:// doi.org/10.1097/00000542200008000-00016

15. Venkatraghavan $L$, Luciano $M$, Manninen P. Anesthetic management of patients undergoing deep brain stimulator insertion. Anesth Analg 2010;110:11381145. https://doi.org/10.1213/ ANE.0b013e3181d2a782

16. Poon C.C.M., Irwin M.G. Anaesthesia for deep brain stimulation and in patients with implanted neurostimulator devices. Br J Anaesth 2009;103:152165. https://doi.org/10.1093/bja/ aep179

17. Khatib R., Ebrahim Z., Rezai A., et al. Perioperative events during deep brain stimulation: the experience at Cleveland clinic. J Neurosurg Anesthesiol 2008;20:3640. https://doi.org/10.1097/ ANA.0b013e318157a15a

18. Granta R., Gruenbaumb S.E., and Gerrarda J. Anaesthesia for deep brain stimulation: a review. Curr Opin Anaesthesiol. 2015 October; 28(5): 505510. https://doi.org/10.1097/ AC0.0000000000000230

19. Rozet I., Muangman S., Vavilala
M.S., et al. Clinical experience with dexmedetomidine for implantation of deep brain stimulators in parkinson's disease. Anesth Analg. 2006; 103:12248. https://doi.org/10.1213/01. ane.0000239331.53085.94

20. Lin N., Vutskits L., Bebawy J.F., Gelb A.W. Perspectives on dexmedetomidine use for neurosurgical patients. J Neurosurg Anesthesiol 2019. https://doi.org/10.1097/ ANA.0000000000000554

21. Hsu Y.W., Cortinez L.I., Robertson K.M., et al. Part I: Dexmedetomidine pharmacodynamics. Anesthesiology 2004;101:1066 -76. https:// doi.org/10.1097/00000542200411000-00005

22. Martínez-Simón A., Alegre M., Honorato-Cia C., et al. Effect ofdexmedetomidine and propofol on basal ganglia activity in Parkinson disease: a controlled clinical trial. Anesthesiology. 2017;126:10331042. https://doi.org/10.1097/ ALN.0000000000001620

23. Chernik D.A., Gillings D., Lane $\mathrm{H}$., et al. Validity and reliability of the observer's assessment of alertness/sedation scale: Study with intravenous midazolam. J Clin Psichopharmacol 1990; 10:244-51 https://doi. org/10.1097/00004714199008000-00003

24. Sewella D. and Smith M. Awake craniotomy: anesthetic considerations based on outcome evidence. Curr Opin Anesthesiol 2019, 32:546552 https://doi.org/10.1097/ ACO.0000000000000750

25. Bekker A., Kaufman B., Samir $\mathrm{H}$., et al. The use of dexmedetomidine infusion for awake craniotomy. Anesth Analg 2001;92:1251-3. https:// doi.org/10.1097/00000539200105000-00031 
26. Fogarty-Mack P., Perrine K., Kobylarz E., et al. Dexmedetomidine and neurocognitive testing in awake craniotomy. J Neurosurg Anesthesiol 2004; $16: 20-5$. https://doi. org/10.1097/00008506200401000-00005
27. Ramsey M.A., Luterman D.L. Dexmedetomidine as a total intravenous anesthestic agent. Anesthesiology 2004; 101:787-90. https:// doi.org/10.1097/00000542200409000-00028

28. Morace R., De Angelis M.,
Aglialoro E., et al. Sedation with alpha-2 agonist dexmedetomidine during unilateral subthalamic nucleus deep brain stimulation: a preliminary report. World Neurosurg. 2016;89:320328. https://doi.org/10.1016/j. wneu.2016.01.037 